\title{
Automated Pose Estimation of Objects Using Multiple ID Devices for Handling and Maintenance Task in Nuclear Fusion Reactor*)
}

\author{
Tomohiro UMETANI, Jun-ichi MORIOKA, Kenji INOUE ${ }^{1)}$, Tatsuo ARAI ${ }^{2)}$, \\ Yasusi $\mathrm{MAE}^{2)}$ and Yuichi TAMURA \\ Konan University, 8-9-1 Okamoto, Higashinada, Kobe 658-8501, Japan \\ 1) Yamagata University, 4-3-16 Jonan, Yonezawa, Yamagata 992-8510, Japan \\ 2) Osaka University, 1-3 Machikaneyama, Toyonaka, Osaka 560-8501, Japan
}

(Received 6 December 2010 / Accepted 29 January 2011)

\begin{abstract}
This paper describes a method for the automated estimation of three-dimensional pose (position and orientation) of objects by autonomous robots, using multiple identification (ID) devices. Our goal is to estimate the object pose for assembly or maintenance tasks in a real nuclear fusion reactor system, with autonomous robots cooperating in a virtual assembly system. The method estimates the three-dimensional pose for autonomous robots. This paper discusses a method of motion generation for ID acquisition using the sensory data acquired by the measurement system attached to the robots and from the environment. Experimental results show the feasibility of the proposed method.
\end{abstract}

(C) 2011 The Japan Society of Plasma Science and Nuclear Fusion Research

Keywords: assembly, virtual assembly tool, data management, pose esetimation, radio frequency identification

DOI: $10.1585 /$ pfr.6.2406079

\section{Introduction}

The integrated management of objects (such as construction materials) using ID devices (such as radio identification (RFID) devices) has been proposed in the area of construction automation [1]. In addition, a virtual assembly system for large-scale devices, for example a nuclear fusion reactor system, has also been proposed [2-4]. Based on the integrated data management of the real and computational world and the virtual assembly system, this requires assembly and maintenance schema for the largescale device using tele-operated autonomous robots [5], as shown in Fig. 1.

It is crucial that the status of object and its information be continuously updated. For example, the system needs to know the object's availability, pose (position and orientation), form, and the task information [6]. For example, the status of the large-scale device can be changed after the device is assembled. The autonomous robot should inspect the environment, and then update the device data for the maintenance task within the virtual assembly system.

This paper describes a method for estimating the three-dimensional pose (position and orientation) of objects by autonomous robots using multiple ID devices. The robot estimates the object pose on the basis of the results acquired from the devices attached to the object. The range measurement system generates the motion of the ID reader attached to the robot.

author'se-mail: umetani@konan-u.ac.jp

*) This article is based on the presentation at the 20th International Toki Conference (ITC20).

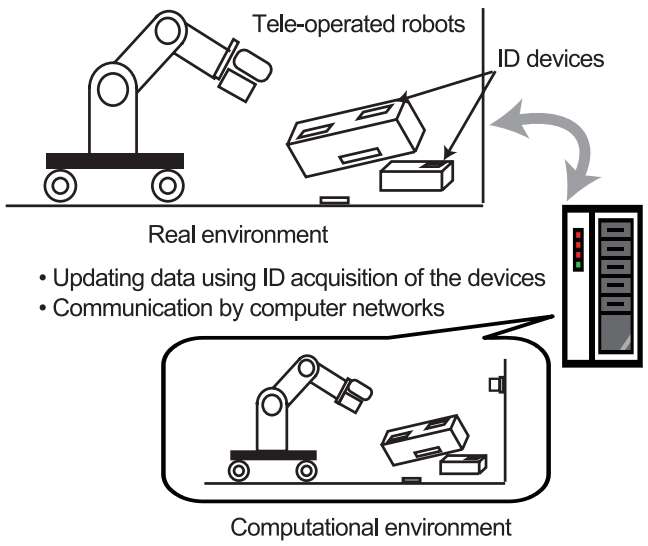

Fig. 1 Integrated data management of an assembly environment and its computational environment using ID devices.

This paper discusses a method of motion generation for ID acquisition using the sensory data acquired by the measurement system attached to the robots and from the environment. The pose estimation method using ID devices has been proposed [7]. In this method, the ID acquisition is conducted by a human operator. A tele-operation system requires motion generation of the robot for ID acquisition.

Our goal is to estimate the object pose for assembly or maintenance tasks in a real nuclear fusion reactor system, with autonomous robots cooperating within a virtual assembly system. In this paper, our experimental testbed is a laboratory room, which is an environment that can evaluate the accuracy of the estimation results. Experimental results show that the proposed method is feasible. 


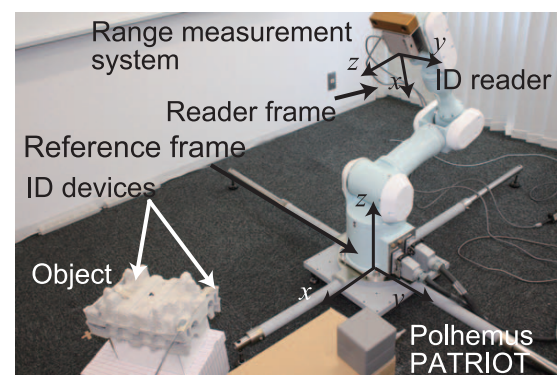

Fig. 2 Overview of experimental environment.

\section{Research Methodology}

This section provides an overview of the experiment and the method of motion generation for ID acquisition using the sensory data of the robot.

\subsection{System overview}

Figure 2 shows the setup of the experiment. We use a Mitsubishi Heavy Industry, Inc., PA-10 as the robot platform for the ID acquisition experiment. The experiment also uses an RFID reader (V680-HS65, OMRON, Japan) and RFID devices (V680-D2KF67 M, OMRON, Japan). The stereo vision system is a PointGrey Research Bumblebee 2 (focal length $f=3.8 \mathrm{~mm}$ ), and is used to obtain the background image from the viewpoint of the robot arm. The RFID reader and stereo vision system are attached to the end of the robot arm. We use a Polhemus PATRIOT (Polhemus, USA), a six degrees of freedom tracking sensor to integrate the coordination frame of the robot and the object in order to evaluate the accuracy of the object pose.

The origin of the reference coordination frame is set to coincide with that of the coordination frame of the robot arm. The $x$-axis of the reference coordination frame is positioned so that it points towards the front of the robot arm, and the $y$-axis of the reference coordination frame points to the left side of the robot arm. The z-axis of the reference coordination frame is set parallel to the vertical direction of the robot arm. The coordination frame of the experimental environment is shown in Fig. 2.

\subsection{Problem setting}

The conditions of the target object are described as follows:

- RFID devices are attached to the upper surface of the object. The robot obtains the IDs of the devices using the RFID reader attached to the object.

- The robot has no preliminary information regarding the positions and orientations of the ID devices with respect to the object coordination frame.

- To simplify the experiment, there are no objects in front of the robot in the initial stage. The robot first obtains the background image, and then an object is set in front of it as the moving object. We assume only one object is present in the environment. Several RFID devices are attached to the object.

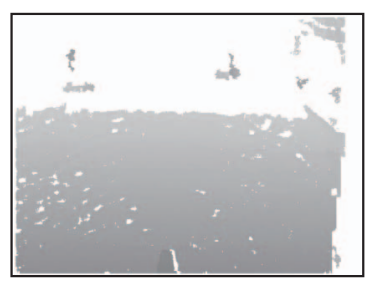

(a) Initial range image

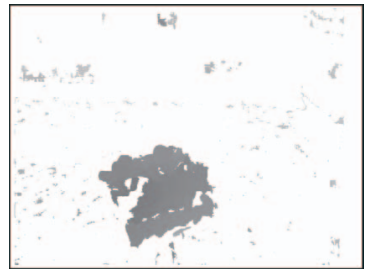

(c) Range image (object area)

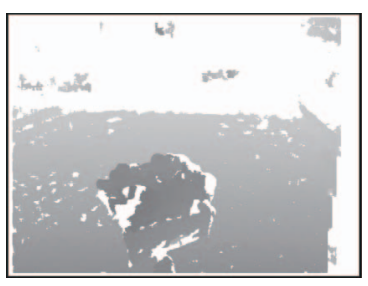

(b) Range image of environment with object

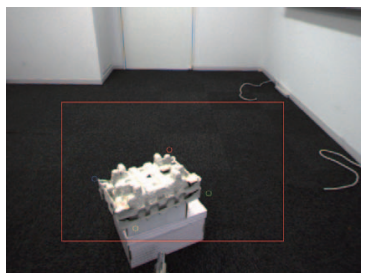

(d) Acquired object area
Fig. 3 Acquired object area of the environment.

- The robot can identify each RFID device by ID acquisition. The position and direction of the RFID device with respect to the object coordination frame are registered beforehand.

\subsection{Motion generation for acquisition of multiple IDs of the devices}

Here, we explain the procedure for generating the controlled robot motion so that it can read multiple IDs of devices in order to acquire the object data:

(1) The robot records the background image of the surrounding environment, which it uses as the range image (Fig. 3 (a)). The thick area of the range image indicates that the area is close to the measurement system, and the pale area indicates that the area is further away.

(2) The robot detects the object using the background subtraction method based on the previously acquired range image (Figs. 3 (b), (c)).

(3) The robot obtains the characteristic points of the moving object from the object surface (Fig. 3 (d)). The red rectangle shown in Fig. 3 (d) indicates the area covered by the ID reader when searching for characteristic points of the data set. These points are defined on the basis of the layout of the experiment.

(4) Based on the characteristic points and by using a controlled motion, the robot reads the IDs of the devices (Fig. 4). The values $\boldsymbol{P}_{R, z \max }, \boldsymbol{P}_{R, x \min }, \boldsymbol{P}_{R, y \text { max }}$, and $\boldsymbol{P}_{R, y \text { min }}$ indicate the object features points, the highest point, the nearest point, the left edge, and right edge of the object area, respectively. These feature points were obtained in step (3).

(5) After the robot acquires the multiple IDs of the devices, it estimates the object pose on the basis of the results of the ID readings.

(6) The robot moves back to the initial position, obtains 


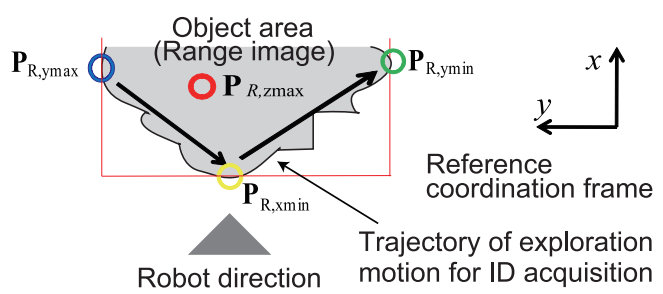

Fig. 4 Characteristic points of the object and trajectory of exploration motion for ID acquisition.

the range image again, and updates the background image of the environment.

In this way, the robot obtains the object data by using the ID devices.

\subsection{Object-pose estimation based on multi- ple ID acquisition}

We estimate the three-dimensional object pose (position and orientation) using the results of ID acquisition. We assume that the ID reader is a small commercial RFID reader, which has a small detection range, and that the object size is much larger than the communication range of the RFID reader. Based on these assumptions, we can describe the properties of the ID acquisition as follows:

- The reader can obtain the ID of the devices placed at the front of the ID reader.

- The ID reader can read the ID device if the device is within the detection range of the ID reader and directed toward the reader.

- The ID reader can read the ID device even if the device rotates about its axis in the direction of the ID reader.

Figure 5 illustrates the geometric model of the ID reader and the device, $D_{i}$, used for acquiring the information. Variables $\boldsymbol{p}_{R, D i}$ and ${ }^{W} R_{R, D i}$ refer to the position and the orientation of the ID reader while acquring the ID of device, $D_{i}$. The reader direction, $\boldsymbol{r}_{D i}$, is the $z$-axis of the reader coordination frame. Variables $\boldsymbol{p}_{c, D i}$ and $R_{\Omega, D i}$ refer to the displacement and the relative orientation of device $D_{i}$ with respect to the reader coordination frame. It is difficult to measure $\boldsymbol{p}_{c, D i}$ and $R_{\Omega, D i}$ directly during ID acquisition. Variables $\boldsymbol{p}_{o, D i}$ and $\boldsymbol{k}_{o, D i}$ refer to the position and the direction of device $D_{i}$ with respect to the object coordination frame. Variables $\boldsymbol{p}_{o b j}$ and ${ }^{W} R_{o b j}$ refer to the position and orientation of the object.

From Fig. 5 and the assumptions outlined earlier, the geometric relation for ID acquisition is given as follows [7]:

$$
\begin{aligned}
& \boldsymbol{p}_{R, D i}+{ }^{W} R_{R, D i} \boldsymbol{p}_{c, D i}-{ }^{W} R_{o b j} \boldsymbol{p}_{o, D i}-\boldsymbol{p}_{o b j}=\mathbf{0}, \\
& \boldsymbol{r}_{D i} \cdot{ }^{W} R_{o b j} R_{\Omega, D i} \boldsymbol{k}_{o, D i}+1=0,
\end{aligned}
$$

where "." denotes the inner product of the vectors. Note that ${ }^{W} R_{o b j}$ is represented by the unit quaternion

$$
\boldsymbol{q}_{o b j}=\left[\begin{array}{llll}
q_{o b j 1} & q_{o b j 2} & q_{o b j 3} & q_{o b j 4}
\end{array}\right]^{T},
$$

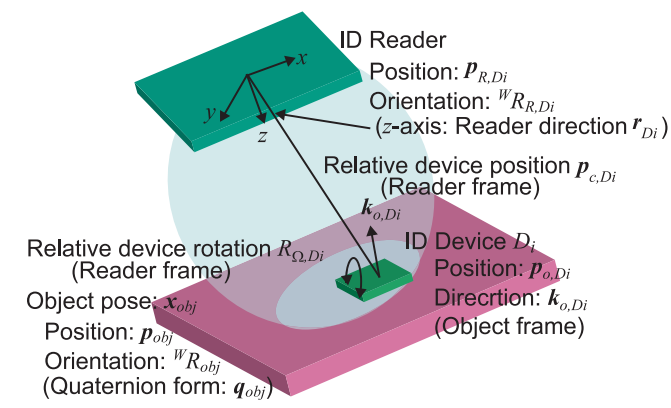

Fig. 5 Geometric model of ID acquisition [7].

where

$$
q_{o b j 1}=\cos \frac{\phi_{o b j}}{2}, \quad \boldsymbol{\epsilon}_{o b j}=\boldsymbol{u}_{o b j} \sin \frac{\phi_{o b j}}{2},
$$

where $\boldsymbol{\epsilon}_{o b j}=\left[\begin{array}{lll}q_{o b j 2} & q_{o b j 3} & q_{o b j 4}\end{array}\right]^{T}$, and $\boldsymbol{u}_{o b j}$ and $\phi_{o b j}$ refer to the equivalent rotation axis and the rotation angle of the object, respectively [8].

We estimate the object pose using the geometric relation of the ID acquisition for multiple ID devices. The object pose, $\boldsymbol{x}_{o b j}=\left[\begin{array}{ll}\boldsymbol{p}_{o b j}^{T} & \boldsymbol{q}_{o b j}^{T}\end{array}\right]^{T}$, is estimated as the solution of the following non-linear programming problem:

$$
J\left(\boldsymbol{x}_{o b j}\right)=\frac{1}{2} \sum_{i=1}^{N} \sum_{j=1}^{4}\left\{f_{i, j}\left(\boldsymbol{x}_{o b j}\right)\right\}^{2} \rightarrow \min
$$

subject to

$$
\begin{aligned}
g\left(\boldsymbol{x}_{o b j}\right)= & \left|\boldsymbol{q}_{o b j}\right|-1=0 \\
h_{i, 1}\left(\boldsymbol{x}_{o b j}\right)= & \left|\boldsymbol{p}_{R, D i}+r_{c, D i} \boldsymbol{r}_{D i}-{ }^{W} R_{o b j} \boldsymbol{p}_{o, D i}-\boldsymbol{p}_{o b j}\right| \\
& -r_{c, D i} \leq 0 \\
h_{i, 2}\left(\boldsymbol{x}_{o b j}\right)= & { }^{W} R_{R, D i} \boldsymbol{r}_{D i} \cdot{ }^{W} R_{o b j} R_{\Omega, D i} \boldsymbol{k}_{o, D i} \\
& -\cos \left(\pi-\theta_{c, D i_{\max }}\right) \leq 0
\end{aligned}
$$

where $J\left(\boldsymbol{x}_{o b j}\right)$ indicates the evaluation function, and $f_{i, j}\left(\boldsymbol{x}_{o b j}\right)$ is the geometric relation for $D_{i}$, corresponding to eqs. (1) and (2). Variables $r_{c, D i}$ and $\theta_{c, D i_{\max }}$ refer to the radius of the communication range of the ID reader and the allowable inclination of the ID acquisition, respectively. Equations (3) and (4) show that device $D_{i}$ is in the communication range of the ID reader.

\section{Experiment}

This section describes an experiment that estimates the object pose to show the feasibility of the proposed method. First, we introduce the experimental conditions, and then, we discuss the experimental results.

\subsection{Experimental setting}

Figure 6 shows the procedure of the pose estimation experiment. The object that we are trying to estimate is compared with the measurement result by the position 


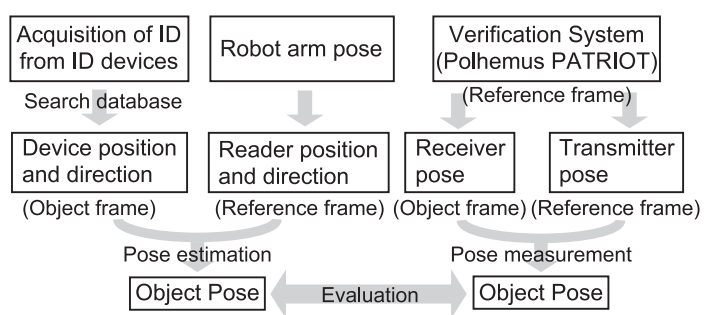

Fig. 6 Procedure of experiment.

Table 1 Layout of ID devices

\begin{tabular}{ccc}
\hline ID & Position $\boldsymbol{p}_{o, D i}[\mathrm{~mm}]$ & Direction $\boldsymbol{k}_{o, D i}$ \\
\hline 1 & {$[170,279,4]^{T}$} & {$[0,0,1]^{T}$} \\
2 & {$[175,14,0]^{T}$} & {$[0,0,1]^{T}$} \\
3 & {$[69,208,32]^{T}$} & {$[0,0,1]^{T}$} \\
\hline
\end{tabular}

measurement system. We set 15 object poses and then estimated the poses on the basis of the result of ID acquisition by the robot. The object was set with various horizontal poses. We used the Polhemus PATRIOT to measure the object pose. The receiver of the measurement system is attached to the origin of the object coordination frame.

Three ID devices were attached to the object, each with a unique ID. Each ID and the position and direction of the device, $\boldsymbol{p}_{o, D i}$ and $\boldsymbol{k}_{o, D i}$, were registered in the data management server in advance, as shown in Table 1. Since the communication range of the reader was 100 [mm], the distance from the ID reader to the device was set to 50 [mm] $\left(\boldsymbol{p}_{c, D i}=\left[\begin{array}{lll}0 & 0 & 50\end{array}\right]^{T}\right)$, and $R_{\Omega, D i}$ was set to the initial matrix. Variables $r_{c, D i}$ and $\theta_{c, D i_{\max }}$ were set to 50 [mm] and 45 [deg], respectively.

\subsection{Results and discussions}

We succeeded in 10 out of 15 trials. Five trials failed. In the successful trials, the robot acquired the ID of the devices between 6 and 21 times using ID acquisition motion and between 2 and 10 times for each device. In the failed cases, ID acquisition failed. The object feature points are measured by the range measurement system. The uncertainty of the feature points was one of the reasons for failure, as the robot generates the trajectory for ID acquisition using these feature points.

The average estimation errors were $5.55[\mathrm{~mm}]$, $11.9[\mathrm{~mm}], 77.1[\mathrm{~mm}]$ for the $x, y$ and $z$-axis, respectively. The average estimation error with respect to distance was $79.1[\mathrm{~mm}]$. The standard deviations for the errors were $7.2[\mathrm{~mm}], 15.8[\mathrm{~mm}], 13.1[\mathrm{~mm}]$ for the $x, y$ and $z$-axis, respectively. The error for the $z$-axis is larger than those for the other axes, because the measurement error for the height obtained by the range measurement system is large. The three-dimensional position and orientation of the object were estimated using the results of acquiring the multiple ID devices using multiple ID readings from the same devices.

We show the feasibility of pose estimation using mul- tiple ID devices. However, the estimation error is quite large for the object size. In future work, we will address the adaptive motion of the ID reader, based on the result of ID acquisition and the exploration motion for ID acquisition.

The experimental results are fundamental, and the target object is set horizontally. The object area should be modeled as a polyhedron to generate the motion for an inclined object and a various shaped object for the virtual assembly system. The improvement of the detection of the object area is also a future work of the study.

\section{Conclusion}

This paper described a method that autonomous robots can use to estimate an object's pose using multiple ID devices in an assembly and maintenance system for a large-scale device, especially a fusion reactor system. In order to successfully integrate the data management of the real and computational world and the virtual assembly system, it is crucial that the status of an object be continuously updated, including its availability and pose (position and orientation).

Here, we described the geometric model of the ID reader and the devices during data acquisition. In addition, we discussed a method for controlling the motion of the ID reader based on the subtraction of the range image of the environment. The robot obtains the IDs of the devices based on the range image of the environment. Experimental results have shown the feasibility of the method.

Future work includes improvements in the effectiveness of using the sensory data of the robot, adaptive motion based on the estimation results, and the data update scheme for the virtual assembly system.

\section{Acknowledgments}

This work was supported in part by a Grant-in-Aid for Scientific Research KAKENHI(22500114), JAPAN, and the Hirao Taro Foundation of the Konan University Association for Academic Research, JAPAN.

[1] J. Yagi, E. Arai and T. Arai, Automat. Constr. 14, 477 (2005).

[2] N. Mizuguchi, Y. Tamura, S. Imagawa, A. Sagara and T. Hayashi, Fusion Eng. Des. 81, 2755 (2006).

[3] Y. Tamura, N. Mizuguchi, S. Matsumoto and H. Ueki, in Proc. 17th Int. Conf. Artificial Reality and Telexistence 284 (2007).

[4] Y. Tamura, A. Kageyama, H. Nakamura, N. Mizuguchi and T. Sato, J. Plasma Phys. 72, 1065 (2006).

[5] F. Inoue, K. Watanabe, A. Wakabayashi and Y. Nekomoto, J. Robotics and Mechatronics 12, 84 (2000).

[6] T. Umetani, T. Arai, Y. Mae, K. Inoue and J. Maeda, Automat. Constr. 15, 777 (2006).

[7] T. Umetani, K. Inoue and T. Arai, Comput.-Aided Civ. Inf. 26, 356 (2011).

[8] J. Funda, R.H. Taylor and R.P. Paul, IEEE T. Robot. Autom. 6, 382 (1990). 